Produto \& Produção, vol. 19, n.1, p.01-13. 2019

RECEBIDO EM 17/11/2018. ACEITO EM 15/01/2019.

\author{
José Carlos Alves Cordeiro \\ Universidade Metodista de Piracicaba, Unimep, SP \\ jccordeiro@unimep.br
}

Silvana Mendes da Silva

Instituto Federal de Educação, Ciência e Tecnologia de São Paulo, IFSP, SP.

smendessilva@uol.com.br

\author{
Adriano Maniçoba da Silva \\ Instituto Federal de Educação, Ciência e Tecnologia de São Paulo, IFSP, SP. \\ adriano_m_s@hotmail.com \\ William de Paula Ferreira \\ Instituto Federal de Educação, Ciência e Tecnologia de São Paulo, IFSP, SP. \\ william.ferreira@ifsp.edu.br
}

\title{
Estratégias híbridas de produção em sistemas make to stock (MTS) e make to order (MTO) com otimização multiobjetivo
}

\begin{abstract}
Resumo
Dentre as estratégias no âmbito da produção e operações, diversas decisões sobre o que, quanto e quando produzir devem ser tomadas para otimizar o desempenho. Neste contexto, a decisão sobre os sistemas make to stock (MTS) e make to order (MTO) é fundamental para auxiliar na estratégia de produção e operações. A escolha dicotômica entre um sistema ou outro pode gerar resultados insatisfatórios no que tange ao atendimento da demanda para diferentes tipos de pedidos. Desta forma, o objetivo deste estudo consiste em propor uma estratégia híbrida de produção em sistemas MTS e MTO com otimização multiobjetivo para que se gere uma programação da produção equilibrada e satisfatória do sob a ótica da demanda. Para tanto, foi realizado um estudo de caso em uma empresa do ramo metalúrgico, situada na região sudeste do Brasil, com sistema aleatório de colocação de pedidos. Por meio dos resultados do modelo de otimização proposto, verifica-se que uma estratégia de produção equilibrada, considerando os sistemas MTS e MTS pode gerar benefícios satisfatórios para os diferentes tipos de pedidos.
\end{abstract}

Palavras-chaves: sistema MTS, sistema MTO, otimização multiobjectivo.

\begin{abstract}
Among the strategies in the field of production and operations, several decisions about what, when and when to produce must be taken to optimize performance. In this context, the decision on make to stock (MTS) and make to order (MTO) systems is critical to assist the production and operations strategy. The dichotomous choice between one system or another can generate unsatisfactory results in terms of meeting demand for different types of orders. In this way, the objective of this study is to propose a hybrid strategy of production in MTS and MTO systems with multiobjective optimization to generate balanced and satisfactory production scheduling from the perspective of demand. For that, a case study was carried out in a metallurgical company located in the southeast region of Brazil, with a
\end{abstract}


random order placement system. By means of the results of the proposed optimization model, a balanced production strategy considering the MTS and MTS systems can generate satisfactory benefits for the different types of orders.

Key words: MTS system, MTO system, multiobjective optimization.

\section{Introdução}

As indústrias brasileiras se encontram em um ambiente de alta competitividade. A busca por melhores resultados econômicos é intensa. Para isso é preciso entender os processos empresariais, a fim de estabelecer melhores estratégias de negócio e conquistar novos mercados consumidores. As estratégias de negócio têm sido direcionadas na busca constante de inovações e respectiva customização dos produtos e serviços. Neste contexto, é de vital importância possuir agilidade nas decisões estratégicas, permitindo que a empresa altere sua forma de competir de acordo com as mudanças do mercado e do contexto econômico.

Dentre as estratégias de negócio, estão as estratégias funcionais de produção que se destacam pelo seu impacto no processo competitivo das empresas, onde as decisões sobre o que, quanto e quando produzir tornam-se questões desafiadoras neste ambiente de constantes mudanças.

A informação e o conhecimento são recursos importantes para que as empresas se mantenham em um ambiente dinâmico e competitivo que, entre outras características, apresentam menor nível de previsibilidade e maior risco. Neste contexto, os processos produtivos devem considerar multicritérios na tomada de decisão tornando esta atividade mais complexa. As decisões no âmbito da produção podem ser baseadas em análises, trade-offs, priorizando a produção customizada, o sistema make to order (MTO), lotes econômicos de produção, ou o sistema make to stock (MTS). A tecnologia da informação com a utilização de banco de dados e o seu relacionamento por meio de softwares interativos propicia um ambiente para que as empresas possam tomar melhores decisões, gerar informações e relacioná-las ao negócio.

A área de operações pode orientar suas decisões de acordo com as escolhas estratégicas da empresa, ou seja, escolher em quais critérios competitivos poderia focar seus recursos operacionais para dar suporte à estratégia competitiva da empresa e, conforme sua atuação ao longo do tempo, transformar as próprias operações em fonte de vantagem competitiva. Nas organizações, gestores a todo o momento são colocados à prova para optar sobre quais são as melhores decisões a serem tomadas no sentido de atender questões como aumento de produtividade, redução de custos e melhorar índices de desempenho de seus indicadores.

Este estudo busca contribuir com o processo de decisões em estratégias de produção no que se refere a decisões de otimização de recursos produtivos. Uma dessas decisões, foco deste trabalho, busca auxiliar na resolução do seguinte problema: Quanto e quais produtos devem ser produzidos de forma a aumentar a produtividade e lucratividade mantendo níveis de serviço competitivos? Uma vez que, não se disponha de uma sistemática para produzir MTO ou MTS, pois estas decisões podem ser pautadas pelo ingresso de pedidos, que pode ser probabilística, é importante planejar a priori qual será a proporção de produção de cada sistema. Desta forma, para estudar a melhor forma de produzir as diferentes proporções de MTO e MTS de forma combinada foi escolhida uma linha de produção de uma empresa metalúrgica localizada na região sudeste do Brasil como objeto de análise.

Cordeiro e Assumpção (2014) sugeriram um modelo com regras heurísticas de classificação para orientar as estratégias MTS e MTO. A partir do modelo apresentado pelos autores, este estudo consiste em propor uma estratégia híbrida de produção em sistemas MTS e MTO com otimização multiobjetivo para que se gere uma programação da produção equilibrada e satisfatória do sob a ótica da demanda. Desta forma, pretende-se demonstrar por meio de uma análise e otimização de recursos produtivos de uma empresa metalúrgica, com produção de diversos tipos de produtos confeccionados em aço, e cujo sistema de entradas de pedidos é aleatório, como as decisões sobre os tipos de produção, MTO ou MTS impactam na produtividade e lucratividade da empresa. Para atingir o objetivo, foi criado um modelo de otimização multiobjetivo que ponderou dois objetivos distintos na sua execução resultando numa estratégia híbrida de produção nos sistemas MTO e MTS. 
Este artigo está organizado da seguinte forma: A seção 2 resgata a revisão da literatura sobre estratégias, estratégias de atendimento MTO e MTS, pesquisa operacional e programação linear; A seção 3 descreve a metodologia e apresenta o estudo de caso, sendo a seção 4 reservada para os resultados e discussões e a seção 5 para as considerações finais.

\section{Estratégia}

Tornar as estratégias delineadas pela alta direção, em ações alinhadas em toda a organização é um desafio para a alta e média gerência sendo que suas ações devem ser orientadas para que as pessoas tomem decisões de acordo com os objetivos e resultados esperados.

Slack, Chambers e Johnston (2007) definiram estratégia como um padrão global de decisões e ações que posicionam a organização em seu ambiente e têm a finalidade de fazê-la atingir seus objetivos de longo prazo.

\subsection{O três níveis estratégicos da empresa}

Paiva, Carvalho Junior e Fensterseifer (2009) apresentam os três níveis estratégicos de uma organização. A estratégia da corporação (grupo empresarial), a estratégia de negócios (unidade estratégica de negócios, empresa ou divisão), que se relaciona com a obtenção e a manutenção da vantagem competitiva, e a estratégia funcional, ligada às diversas áreas da unidade estratégica de negócios, como a estratégia de manufatura ou operações, tema deste trabalho.

O nível das estratégias funcionais contribui para atender os objetivos estratégicos no negócio, traduzir os objetivos competitivos do negócio em objetivos funcionais, gerenciarem os recursos das funções de forma a atingir os objetivos funcionais e estabelecer prioridades de melhoria de desempenho (SLACK; CHAMBERS; JOHNSTON, 2007). São influenciadas pelas expectativas da alta direção a respeito da função, habilidades do pessoal, capacitação tecnológica e sua organização e planejamento.

Jabbour e Alves Filho (2010) demonstraram que trabalhos sobre estratégias de produção mais recorrentes estão relacionados ao conteúdo, isto é, representam as decisões tomadas pela corporação para eficácia da estratégia. Trabalhos sobre formulação e implementação estão representados em menor número. Os autores destacaram ainda em sua pesquisa, a relevância de estudos acadêmicos e econômicos na área de estratégia ao reforçarem quão importante é o papel da produção no desempenho de empresas pertencentes a um setor com relevância na indústria nacional e, ainda, como são úteis e importantes na tomada de decisão os conceitos desenvolvidos no campo teórico da estratégia de produção e atribuem os avanços nesse campo a interação entre teoria e prática.

Os gestores devem traduzir para operações as definições estratégicas corporativas ou das unidades de negócio. Por exemplo, definições estratégicas como a busca do retorno sobre investimentos podem não corresponder a ações específicas para o chão de fábrica. É necessário identificar medidas na gestão de operações que impactam no processo estratégico da empresa. Novas tecnologias de operações tornam possível maior flexibilidade em projetos e mix de produtos, respostas rápidas às mudanças de mercado, mais informações e maior agilidade na programação da produção.

Paiva, Carvalho Junior e Fensterseifer (2009) admitem que exista certa concordância que as definições sobre estratégias de produção para fazer coincidir com os objetivos da empresa ou unidade de negócios, de forma a alcançarem os objetivos da área de operações, buscarem vantagens competitivas e focalizar um padrão de decisões consistente.

Com intuito de direcionar alguns objetivos da operação e relacioná-las com a estratégia de negócios, Hopp e Spearman (2000), apresentaram uma hierarquia de objetivos da área de produção como demonstra o esquema da Figura 1. 


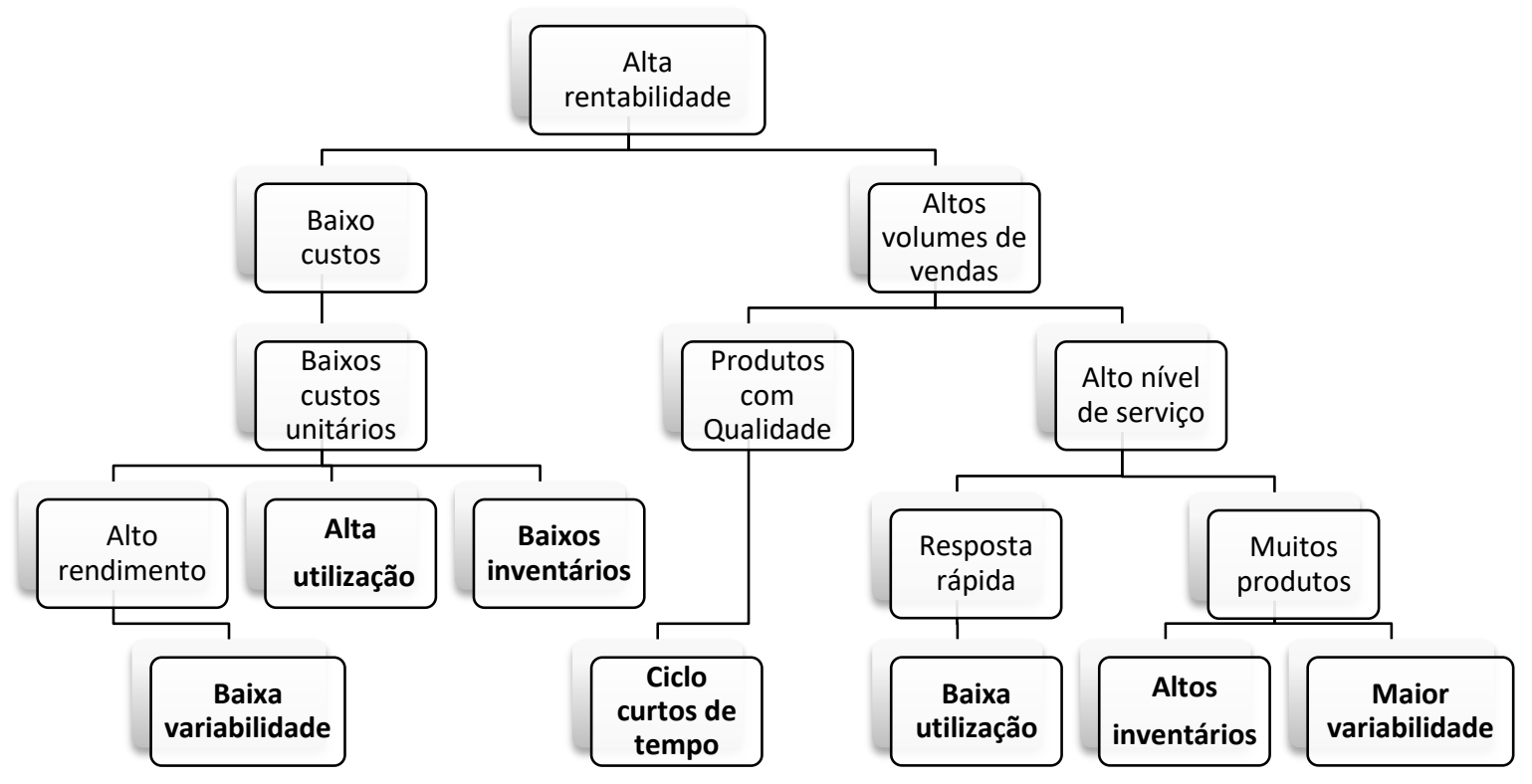

Figura 1 - Hierarquia dos objetivos da mamufatura Fonte: Adaptado de Hopp e Spearman (2000)

$\mathrm{O}$ alto rendimento, a quantidade vendida de produto por unidade de tempo, inventário (estoques) e custos operacionais são objetivos que ligam o desempenho da operação com as estratégias de negócio.

\subsection{Estratégias de produção e sistemas MTS e MTO}

As áreas de decisão da estratégia de manufatura são divididas em estruturais e infra estruturais, sendo que as estruturais podem ser referentes aos investimentos de longo prazo, como por exemplo, instalações físicas e equipamentos, consideradas como mais estratégicas. As infra estruturais descrevem sistemas, políticas e práticas que determinam como os aspectos estruturais da organização são gerenciados, e são consideradas como decisões táticas (SLACK; CHAMBERS; JOHNSTON, 2007).

Os critérios de competição ou prioridades competitivas têm sido propostos na literatura e temse alterado ao longo do tempo, entre os mais recentes são: qualidade, velocidade, confiabilidade de entrega, flexibilidade e custo (PAIVA; CARVALHO JUNIOR; FENSTERSEIFER, 2009).

Alves, Nogueira e Bento (2011) afirmaram que as prioridades competitivas de produção são definidas, então, em função das estratégias competitivas (que refletem fatores de mercado como, por exemplo, demanda em maior ou menor nível) e das características das áreas de decisão (que refletem os recursos acumulados e as pressões decorrentes de determinada trajetória). Também observaram que mudanças nas prioridades competitivas de produção (e nas estratégias competitivas e de entrada, eventualmente) e as correspondentes medidas implementadas nas áreas de decisão compõem as estratégias de produção e, ao longo do tempo, marcam os movimentos e as trajetórias das estratégias adotadas pelas empresas.

Uma empresa pode estabelecer estratégias globais ou individuais para sua linha de produtos em função do mercado que se quer competir. Dependendo da estratégia adotada a empresa depara-se com restrições operacionais como prazos para entrega, custos, capacidades produtivas, etc. Por isso, 
frequentemente é necessário tomar decisões que permitam estabelecer um determinado nível de serviço, que leve em conta as restrições dos recursos operacionais da empresa e que atendam os interesses dos clientes e acionistas.

O setor de operações pode contribuir para que a empresa possa manter-se competitiva no mercado tendo uma estratégia de produção flexível, baixos custos e ter rapidez em seus processos. Para tanto é necessário que tenha um planejamento adequado e um processo de tomada de decisão ágil.

Como destacam Guimarães (2010) e Fávero e Zoucas (2016), existem vários paradigmas estratégicos de gestão da manufatura. São modelos estratégicos e integrados de gestão, direcionados a certa situação de mercado que se propõem auxiliar as empresas a alcançarem determinados objetivos de desempenho.

Pode-se ainda destacar que a função produção pode contribuir para melhor atender ao cliente, tendo flexibilidade e rapidez para decidir em produzir para estoque ou MTS ou produzir sob encomenda MTO. Dada a amplitude de produtos e buscando maximizar a utilização da capacidade de seus recursos operacionais, as empresas têm adotado sistemas combinados MTS-MTO, chamados híbridos, para melhor alinhamento das estratégias de produção com as empresariais.

Kaminsky e Kaya (2006) ressaltaram que pressões originadas pela crescente competitividade estão levando as empresas a uma maior ênfase no atendimento ao cliente. Um elemento importante do serviço ao cliente é utilizar o sistema MTS para ter itens em estoque, e também o MTO para reduzir custos de estocagem. Diversas empresas vêm se destacando por optar por uma abordagem híbrida, utilizando sistemas combinados MTS-MTO. Wanrooij (2012) afirma que a combinação de estratégias MTO e MTS traz complexidades no que diz respeito ao planejamento da capacidade, alocação e programação de decisões operacionais e de controle.

Ainda de acordo com Wanrooij (2012) uma estratégia pura de MTO é tipicamente quando os produtos são especificados pelo cliente e consequentemente apresentam um custo maior. Desta forma, pode haver uma variedade maior de produtos. A produção somente é iniciada quando existe uma ordem para sua produção. Portanto, trata-se de um produto com baixa previsibilidade, baixo custo de armazenagem e são "puxados" pelos clientes. Wanrooij (2012) também destaca que, neste sistema, o planejamento da produção é focado na ordem para produção e sua prioridade competitiva são prazos de entrega curtos e confiáveis. Também de acordo com o autor, uma estratégia MTS para produtos que não possuem maior variedade e baixo custo, pode implicar em custo maior de inventários.

Kalantari, Rabbani e Ebadian (2010) apresentam um modelo matemático como objetivo de dar suporte para decisões de aceite ou rejeição de modelos híbridos de produção MTS/MTO em relação a sistemas com limitações de recursos e atribuição de valor diferentes aos clientes. No estudo dos autores observa-se que são levados em consideração na tomada de decisão sobre o aceite ou rejeição de pedidos os atributos quantitativos (preço, prazos de entregas) e qualitativos (importância do cliente).

\subsection{Pesquisa operacional e programação linear}

De acordo com Shamblin (1979), a pesquisa operacional (PO), originalmente desenvolvida na Segunda Guerra Mundial para resolver problemas estratégicos e táticos militares, foi adotada pelas organizações industriais e, com a evolução dos computadores, tornou-se um tipo de abordagem comum na solução de problemas organizacionais.

Segundo Arenales et al. (2015) a pesquisa operacional é uma abordagem científica para auxiliar no processo de tomada de decisões, que procura determinar como melhor projetar, planejar e operar sistemas, usualmente sob condições que requerem alocações eficientes de recursos escassos.

No ambiente comercial competitivo atual, é cada vez mais importante garantir que os recursos limitados de uma empresa sejam usados de maneira mais eficiente possível. Normalmente, isso envolve determinar como alocar os recursos de maneira a maximizar os lucros ou minimizar os custos (RAGSDALE, 2009).

A Programação Linear (PL) visa encontrar a melhor solução para problemas que tenham seus modelos representados por expressões lineares. À sua grande aplicabilidade e simplicidade devem-se a linearidade do modelo. A tarefa da PL consiste na maximização ou minimização de uma função linear, 
denominada função objetivo, respeitando-se um sistema linear de igualdades ou desigualdades, que recebem o nome de Restrições do Modelo (MARINS, 2011).

Lachtermacher (2009) destacou que, em função da necessidade de maximizar ou minimizar uma quantidade (lucro, custo, receita, número de produtos, entre outros), chamada de objetivo, que depende de um ou mais recursos escassos, os processos de otimização de recursos são aplicados a diversas áreas como: determinação do mix de produtos, escalonamento da produção, roteamento e logística, planejamento financeiro, carteira de investimento, análise de projetos, alocação de recursos de mídia, designação de equipes. Um problema de otimização de produção pode ser descrito no seguinte formato geral:

Existindo " $m$ " recursos usados na produção de " $n$ " produtos

$c_{j}$ : lucro na venda de uma unidade do produto $\mathrm{j}=1,2, \ldots, \mathrm{n}$;

$\mathrm{b}_{\mathrm{i}}$ : quantidade disponível do recurso $\mathrm{i}=1,2, \ldots, \mathrm{m}$;

$\mathrm{a}_{\mathrm{ij}}$ : quantidade do recurso $\mathrm{i}$ usada para produzir uma unidade do produto $\mathrm{j}$.

$\mathrm{x}_{\mathrm{j}}$ : quantidade a produzir do produto $\mathrm{j}$ (variáveis de decisão).

Função objetivo Max $\mathrm{Z}=\sum_{j=1}^{n} c_{j} x_{j}$

Restrições sujeito à:

$$
\begin{aligned}
& \mathrm{a}_{11} \mathrm{x}_{1}++\ldots+\mathrm{a}_{\mathrm{n} n} \mathrm{x}_{\mathrm{n}} \leq \mathrm{b}_{1} \\
& \mathrm{a}_{21} \mathrm{x}_{1}++\ldots+\mathrm{a}_{2 \mathrm{n}} \mathrm{x}_{\mathrm{n}} \leq \mathrm{b}_{2} \\
& \mathrm{a}_{\mathrm{m} 1} \mathrm{x}_{1}+\ldots+\mathrm{a}_{\mathrm{mn}} \mathrm{x}_{\mathrm{n}} \leq \mathrm{b}_{\mathrm{m}} \\
& \mathrm{x}_{\mathrm{j}} \geq 0, \mathrm{j}=1,2, \ldots, \mathrm{n}
\end{aligned}
$$

Miguel (2012) destaca que é possível construir modelos que expliquem pelo menos parte do comportamento de processos reais, ou que seja possível capturar pelo menos parte de problemas de tomada de decisão encontrados em processos reais. Pio, Silva e Kawamoto Júnior (2018) utilizaram a programação linear para estudar processos de produção com sistemas puxados. Dassan et al. (2016) otimizaram a programação da produção de uma indústria de placas eletrônicas com a otimização multiobjetivo.

\section{Metodologia}

Para atingir o objetivo delineado de propor uma estratégia híbrida de produção com a utilização da otimização multiobjetivo, foi analisado um estudo de caso único, baseado numa empresa de grande porte de corte e distribuição de bobinas de aço que trabalha com ambientes híbridos de produção.

Trata-se de um estudo de caso utilizando a abordagem qualitativa e quantitativa. Apresentamse os resultados através da resolução do problema de programação linear. Os pesquisadores exerceram a função de observadores diretos, auxiliando na análise final dos dados.

Conforme Martins (2008) destaca, estudo de caso é uma estratégia metodológica de se fazer pesquisa nas ciências sociais. Trata-se de uma metodologia aplicada para avaliar ou descrever situações dinâmicas em que o elemento humano está presente. Busca-se aprender a totalidade de uma situação, descrever, compreender e interpretar a complexidade de um caso concreto, mediante a análise de um objeto delimitado.

Yin (2001) prescreve táticas diferenciadas para cada tipo de estudo de caso. Em relação ao estudo de caso único, o autor o prescreve quando este representa o caso decisivo para testar uma teoria bem formulada, seja para confirma-la, seja para contesta-la, seja ainda para estender a teoria. Esse último, é a finalidade desse estudo pois pretende-se, a partir dessa análise, que ela possa ser estendida a outras empresas que encontrem o mesmo tipo de problema: definir entre produzir para estoque ou para cliente. 


\subsection{A Empresa}

Analisou-se neste estudo uma empresa de corte e distribuição de bobinas de aço. As bobinas são especificadas com pesos de 8 a 14 toneladas e podem ter diversos tipos de especificações (espessura, largura, norma, acabamento, etc.). Num dos processos, objeto de estudo deste trabalho, a empresa executa cortes transversais à largura da bobina em tamanhos que variam de 1.000 a 12.000 $\mathrm{mm}$ de comprimento de acordo com as necessidades do cliente e respectiva capacidade do equipamento. O processo tem como produto final chapas de aço comercializadas pela empresa para diversos tipos de mercados consumidores que utilizam em seus processos internos ou revendem para outros mercados.

A empresas possui em seu estoque itens padrão MTS, que podem ter, por exemplo, comprimentos de $2.000 ; 3.000 ; 6.000 \mathrm{~mm}$, mas também pode comercializar seus produtos nos mais diversos tamanhos, conforme sua necessidade e que pode variar sob o sistema MTO.

O mercado consumidor deste produto tem como característica alta competitividade e seus principais critérios são preço, disponibilidade, prazo e confiabilidade. Consequentemente este tipo de produto oferece baixa margem de lucro e sua produção deve ser feita em larga escala. A empresa organiza seu sistema produtivo com estratégias de atendimento do tipo: produção sob encomenda ou MTO, neste caso a produção é direcionada por um pedido de um cliente. Outra modalidade é a produção para estoque MTS que, neste caso, é programada a partir de análises estatísticas dos produtos comercializados.

No caso de produção sob o sistema MTO, os prazos de entrega são curtos, isto é, para ser competitivo é necessário estar dentro do prazo de entrega mínimo que as empresas concorrentes operam. Na produção MTS, os prazos de entrega são quase que imediatos, e o desempenho depende da disponibilidade em estoque.

A escolha para atender uma estratégia de produção normalmente afeta o desempenho da outra. Quando decide-se atender sob MTO, consequentemente o MTS pode ser afetado. Geralmente o gestor de produção opta por priorizar o atendimento dos produtos MTO em detrimento do MTS por já haver pedido confirmado. Porém, nessa decisão não estão sendo avaliados os resultados quanto à lucratividade e produtividade, que é o que esse estudo propõe elucidar com a otimização multiobjetivo.

Foi constatado que, na empresa analisada, o recurso produtivo estava no limite de sua capacidade de produção e disponibilidade (turnos e horas extras). Alternativas como, por exemplo, investimentos (aquisição de novos equipamentos) ou subcontratação da produção com terceiros não poderiam ser considerados como opção para solução do problema principalmente devido às baixas margens do produto. A figura 2 apresenta um fluxo simplificado do processo:

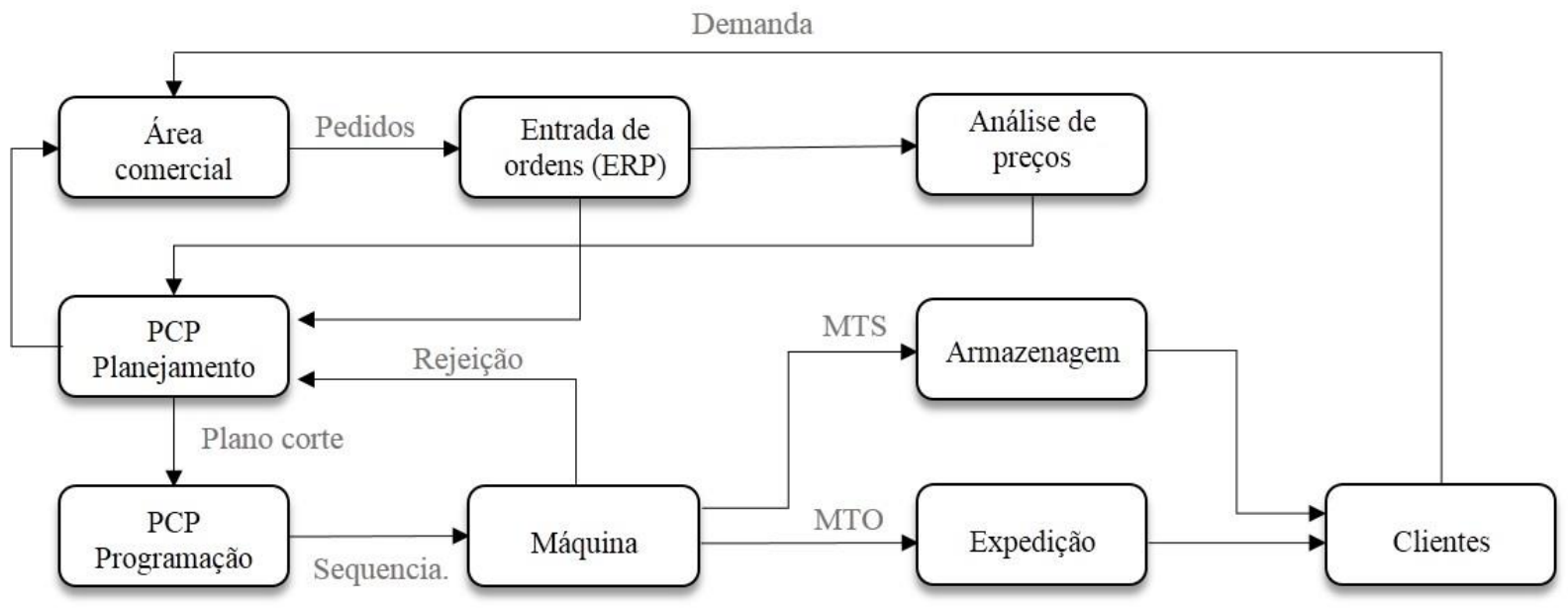

Figura 2 - Macro Fluco do proceso

Fonte: Cordeiro e Assumpção (2014, p. 118) 
A seguinte sequência descreve como o processo de entrada de pedidos ocorre dentro da empresa:

i. Captação/Recebimento dos pedidos junto aos clientes;

ii. Análise e confirmação do pedido em função dos prazos disponíveis;

iii. Digitação do pedido no sistema ERP baseado na tabela de prazos. Não existe restrições no sistema quanto à limitação de entrada de pedidos, poderão ocorrer digitação em excesso ou falta em relação à capacidade do processo. $\mathrm{O}$ sistema permite que a digitação possa ocorrer simultaneamente, e sua sequência somente é determinada quando ocorre a confirmação do pedido, após a digitação.

iv. Liberação do pedido com relação ao preço negociado quando este estiver fora das condições comerciais estabelecidas;

v. Análise dos pedidos, avaliando as condições de processo, especificação e prazos;

vi. Caso exista alguma irregularidade o pedido retorna para a condição (ii) para ajuste sobre a nova tabela;

vii. Com o pedido nas condições regulares é feito o planejamento do corte, roteiro e agrupamento quando as regras estabelecidas para liberação para produção estiverem de acordo;

viii. Com o planejamento do corte e roteiro estabelecidos, os pedidos são analisados e programados conforme regras de prioridade (prazos) e exceções;

ix. O corte é realizado em máquina conforme condições pré-estabelecidas e sequência programada;

X. O material é programado para produção;

xi. O material pode ser faturado para o cliente ou transferido para algum estoque (Centros de Distribuição)

A empresa possui dificuldades quanto à definição de prazos de entrega para seus clientes e consequentemente cumprir este compromisso em função da dificuldade de medir o tempo do ciclo de produção. Atualmente para definição dos prazos de entrega considera-se o total de pedidos sobre a capacidade de produção diária do equipamento mais o tempo estimado de processamento de pedidos.

\subsection{Construção do Modelo}

Na solução de problemas reais dificilmente é utilizada uma única metodologia, mas é comum encontrar um conjunto de duas ou mais formas de solucionar um problema.

Para a solução do problema do caso em estudo foram adotadas abordagens distintas, mas complementares:

- Para classificação MTS e MTO foram utilizadas ferramentas estatísticas, principalmente o conceito do coeficiente de variação.

- Para o problema do mix de produção utilizou-se a otimização multiobjetivo.

\subsection{Levantamento e Coleta de Dados}

O levantamento dos dados foi obtido com informações pela coleta de índices, relatórios e demais dados secundários das áreas relacionadas com o objeto em estudo. O período considerado para análise foi de 39 semanas como amostra do processo.

Algumas comparações foram feitas de forma qualitativa, como a percepção de atendimento e dificuldades de processamento e outras de forma quantitativa, como margem do produto, tempo de setup, perdas e tempo de produção. As informações levantadas são apresentadas na Tabela 1. 
Tabela 1: Levantamento de dados.

\begin{tabular}{|c|c|c|}
\hline Fonte & Análise da informação & Objetivos \\
\hline $\begin{array}{l}\text { Base de entrada de } \\
\text { pedidos }\end{array}$ & $\begin{array}{l}\text { Média, desvio padrão, coeficiente de } \\
\text { variação, tipo dispersão, normalidade. }\end{array}$ & $\begin{array}{l}\text { Avaliar a dispersão de entrada dos pedidos, padrões } \\
\text { de comportamentos e previsibilidades. }\end{array}$ \\
\hline $\begin{array}{l}\text { Entrada de pedidos } \\
\text { no PPCP }\end{array}$ & Analise do processo de programação. & $\begin{array}{l}\text { Verificar as variáveis de programação que } \\
\text { interferem no processo. }\end{array}$ \\
\hline $\begin{array}{l}\text { Planejamento e } \\
\text { sequenciamento }\end{array}$ & $\begin{array}{l}\text { Analise do processo de planejamento e } \\
\text { sequenciamento. }\end{array}$ & $\begin{array}{c}\text { Verificar as variáveis de planejamento que } \\
\text { interferem no processo (ex: agrupamento e métodos } \\
\text { de planejamento). }\end{array}$ \\
\hline $\begin{array}{l}\text { Capacidades do } \\
\text { equipamento }\end{array}$ & Análise da capacidade. & Identificar as restrições. \\
\hline $\begin{array}{l}\text { Histórico de } \\
\text { produção }\end{array}$ & $\begin{array}{l}\text { Média, desvio padrão, coeficiente de } \\
\text { variação, tipo dispersão, normalidade. }\end{array}$ & $\begin{array}{c}\text { Avaliar a dispersão de entrada dos pedidos, padrões } \\
\text { de comportamentos e previsibilidades e comparar } \\
\text { com a capacidade. }\end{array}$ \\
\hline $\begin{array}{l}\text { Histórico de } \\
\text { faturamento }\end{array}$ & $\begin{array}{l}\text { Análise dos volumes de faturamento do } \\
\text { produto estudado. }\end{array}$ & $\begin{array}{l}\text { Avaliar o percentual de faturamento por tipo de } \\
\text { produto - MTO e MTS. }\end{array}$ \\
\hline Histórico de paradas & $\begin{array}{l}\text { Análise das principais paradas que interferem } \\
\text { no processo. }\end{array}$ & Quantificar a interferência das paradas no processo. \\
\hline $\begin{array}{l}\text { Portfólio de } \\
\text { produtos MTS }\end{array}$ & Analise dos volumes e produtos. & $\begin{array}{c}\text { Avaliar o volume do MTS e interferência na } \\
\text { capacidade de produção. }\end{array}$ \\
\hline
\end{tabular}

Fonte: elaborado pelos autores

\subsection{Tratamento Estatístico de Dados}

Em sistemas de manufatura, existem muitos atributos em que variabilidade é de interesse: dimensões físicas, tempos de processo, quebra da máquina, manutenção, qualidade, medidas, temperaturas, dureza do material, tempos de setup, são exemplos de elementos propensos a não uniformidade.

Para análise dos dados foram estabelecidos os elementos que influenciavam no resultado do objeto em estudo. Foram consideradas as seguintes características: a espessura, o comprimento da peça e o tamanho do lote. Para segregar os itens de maior representatividade no processo foi utilizada a regra de Pareto.

Para este estudo foi considerado um intervalo de 39 semanas como período de amostragem e foram calculados média, desvio padrão, coeficiente de variação, frequência do item e lote mínimo conforme destaca a Tabela 2.

Hopp e Spearman (2000) utilizam o coeficiente de variação para representar e analisar variabilidades nos sistemas de produção e consideram que taxas com coeficiente de variação menor que 1,33 possuem uma moderada variação no processo sendo que neste estudo foi considerado um coeficiente menor que 1 . 
Tabela 2: Análise estatística para segregação de dados (PARETO)

\begin{tabular}{c|c|c|c|c|c|c}
\hline $\begin{array}{c}\text { Variáveis / } \\
\text { Características }\end{array}$ & $\begin{array}{c}\text { Valor } \\
\text { médio }\end{array}$ & $\begin{array}{c}\text { Coeficiente } \\
\text { de variação }\end{array}$ & $\begin{array}{c}\text { Frequência } \\
\text { do item }\end{array}$ & $\begin{array}{c}\text { Total da } \\
\text { amostra }\end{array}$ & Itens & Representatividade \\
\hline Espessura & $\begin{array}{c}\text { Maior que } \\
10\end{array}$ & $\begin{array}{c}\text { Menor que } \\
1,0\end{array}$ & $\begin{array}{c}\text { Maior que } \\
32\end{array}$ & 46 & 9 & $98 \%$ \\
\hline Comprimento & $\begin{array}{c}\text { Maior que } \\
10\end{array}$ & $\begin{array}{c}\text { Menor que } \\
1,0\end{array}$ & $\begin{array}{c}\text { Maior que } \\
32\end{array}$ & 259 & 4 & $89 \%$ \\
\hline $\begin{array}{c}\text { Faixa de } \\
\text { peso/lote }\end{array}$ & $\begin{array}{c}\text { Maior que } \\
10\end{array}$ & $\begin{array}{c}\text { Menor que } \\
1,0\end{array}$ & $\begin{array}{c}\text { Maior que } \\
32\end{array}$ & 44 & 8 & $81 \%$ \\
\hline
\end{tabular}

Fonte: Adaptado de Cordeiro e Assumpção (2014, p. 121)

Para definir quais itens seriam considerados MTS e MTO, baseado no coeficiente de variação, frequência relativa e lote mínimo, foram estabelecidos os seguintes critérios conforme a Tabela 3:

Tabela 3: Categorização para análise de MTS e MTO

\begin{tabular}{l|l|c|c}
\hline \multicolumn{1}{c|}{ Frequência } & Coeficiente de variação & Média de saída $>=\mathbf{1 2}$ & Média de saída $<\mathbf{1 2}$ \\
\hline \multirow{2}{*}{$\begin{array}{l}\text { Itens com 12 ou mais } \\
\text { meses de saída }\end{array}$} & Coeficiente de variação $>1$ & $\mathrm{G} 2$ & $\mathrm{G} 6$ \\
\cline { 2 - 4 } & Coeficiente de variação <=1 & $\mathrm{G} 1$ & $\mathrm{G} 4$ \\
\hline $\begin{array}{l}\text { Itens com menos de 12 } \\
\text { meses de saída }\end{array}$ & Coeficiente de variação $>1$ & $\mathrm{G} 5$ & $\mathrm{G} 8$ \\
\cline { 2 - 4 } & Coeficiente de variação <=1 & $\mathrm{G} 3$ & $\mathrm{G} 7$ \\
\hline
\end{tabular}

Fonte: Adaptado de Cordeiro e Assumpção (2014, p. 122)

Onde:

G1 - Considerado baixo risco, indica boa frequência, boa média e baixa variabilidade

G2 - Considerado risco moderado, boa saída, boa média e alta variabilidade.

G3 - Considerado risco moderado, boa saída, boa média e baixa variabilidade.

G4 - Considerado risco moderado, boa saída, baixa média e baixa variabilidade.

G5 - Considerado risco alto, baixa saída, boa média e alta variabilidade.

G6 - Considerado risco alto, boa saída, baixa média e alta variabilidade.

G7 - Considerado risco alto, boa saída, baixa média e baixa variabilidade.

G8 - Considerado risco altíssimo, baixa saída, baixa média e alta variabilidade.

Tanto os dados de frequência como a média de saída poderão ser alterados conforme condições de mercado e fornecedores de matéria prima.

Como a base do sistema MTS é a formação de estoques, é necessário que se incorra em certo risco mínimo em manter capital imobilizado em estoque. Desta forma, forma considerados MTS os materiais dos grupos: G1, G2, G3 e G4 por apresentarem menor risco de não serem comercializados.

Os itens não classificados como MTS foram atribuídos ao grupo de MTO. 


\section{Resultados e Discussão}

Para analisar a estratégia de produção da empresa em relação ao sistema MTO e MTS, foram coletados os dados de capacidade de produção que variavam de um mês para outro devido às diferenças de dias úteis, feriados, manutenções e treinamentos, sendo que foi considerado uma semana com 117,5 horas referente a disponibilidade de produção.

Para obtenção da função objetivo foram analisados os preços médios no período analisado, janeiro a julho de 2015 , e seus respectivos custos onde obteve-se margem líquida de $\mathrm{R} \$ 88,00$ por tonelada para produtos MTO e R $\$ 43,00$ por tonelada para produtos MTS. Os tempos médios de produção para cada tonelada para produtos MTO e MTS foram respectivamente 0,16 horas e 0,13 horas. A Tabela 4 apresenta um resumo dos dados utilizados na modelagem.

Tabela 4: Resumo de dados para modelagem

\begin{tabular}{c|c|c|c}
\hline Dados & MTO & MTS & Base de informação \\
\hline Disponibilidade de produção semanal (horas) & \multicolumn{2}{|c|}{117,5} & Capacidade do equipamento \\
\hline Tempo de produção para 1 tonelada & $\mathbf{0 , 1 6}$ & $\mathbf{0 , 1 3}$ & Histórico de produção \\
\hline Lucro operacional (R\$) por tonelada & $\mathbf{8 8 , 0 0}$ & $\mathbf{4 3 , 0 0}$ & Relatórios operacionais \\
\hline Volume de produção & $\mathbf{7 5 \%}$ & $\mathbf{2 5 \%}$ & Histórico de produção \\
\hline
\end{tabular}

Fonte: elaborado pelos autores

Para o processo de modelagem foram consideradas as seguintes variáveis de decisão, restrições e funções objetivo:

Variáveis de decisão: $\quad \mathrm{x}_{1}$ - Produção diária dos produtos no sistema MTO

$\mathrm{x}_{2}$ - Produção diária dos produtos no sistema MTS

Restrições:

$0,16 \mathrm{x}_{1}+0,13 \mathrm{x}_{2} \leq 117,5$ ( Limitação semanal do recurso) :

$\mathrm{x}_{1} \geq 0, \mathrm{x}_{2} \geq 0$ (Não - negatividade )

Função objetivo do lucro: Max $L=88 \mathrm{x}_{1}+43 \mathrm{x}_{2}$

Função objetivo da produção: $\operatorname{Max} Z=x_{1}+x_{2}$

Modelo da otimização multiobjetivo:

Min: $Q$

Sujeito a:

$\sum a_{j} x_{i} \leq b j$

$$
\begin{aligned}
& w_{1}\left[64.625-\left(\sum_{i=1}^{2} m_{i} x_{i}\right)\right] / 64.625 \leq Q \\
& w_{2}\left[903-\left(\sum_{i=1}^{2} x_{i}\right)\right] / 903 \leq Q
\end{aligned}
$$

$x_{i} \geq 0$ 
Utilizando o solver do Excel, inicialmente foram executados dois modelos, 1 e 2, com o objetivo de maximizar o lucro (modelo 1) e a produção (modelo 2). Conforme pode ser verificado na Tabela 1, os resultados destes modelos geraram diferentes valores de lucro, participação MTS, participação MTO, foram executados os dois modelos onde obteve-se os seguintes resultados, conforme Tabela 5. Pode-se verificar que os resultados dos modelos sugerem a adoção estrita de um sistema em detrimento de outro. Com base nestes resultados foi conduzida a otimização multiobjectivo (modelo 3) cujo resultado balanceou o lucro e a produção nos dois sistemas.

Tabela 5: Resultados dos modelos

\begin{tabular}{|c|c|c|c|c|c|c|}
\hline Modelo & $\begin{array}{c}\text { Lucro } \\
(\mathrm{R} \$)\end{array}$ & $\begin{array}{c}\text { Participação } \\
\text { MTS (\%) }\end{array}$ & $\begin{array}{c}\text { Participação } \\
\text { MTO (\%) }\end{array}$ & $\begin{array}{c}\text { Volume de } \\
\text { produção/sem. } \\
\text { MTS (t) }\end{array}$ & $\begin{array}{c}\text { Volume de } \\
\text { produção/ sem. } \\
\text { MTO (t) }\end{array}$ & $\begin{array}{c}\text { Volume de } \\
\text { produção/sem. } \\
\text { Total (t) }\end{array}$ \\
\hline 1 & $64.625,00$ & 0 & 100 & 0 & 734 & 734 \\
\hline 2 & $38.865,38$ & 100 & 0 & 903 & 0 & 903 \\
\hline 3 & $56.384,49$ & 37 & 63 & 289 & 499 & 788 \\
\hline
\end{tabular}

Fonte: elaborado pelos autores

Os resultados da otimização multiobjectivo permitem propor uma estratégia híbrida de produção pelo sistema MTO e MTS. Tal como destacam Jabbour e Alves Filho (2010), é importante o alinhamento entre as prioridades competitivas e a estratégia competitiva da empresa para melhorar o desempenho organizacional e também o direcionamento na implementação de práticas de produção na orientação das áreas de decisão. Desta forma, verifica-se que esta proposição de estratégia híbrida suporta o alinhamento entre prioridades competitivas de custo e de atendimento ao consumidor.

Alves, Nogueira e Bento (2011) também afirmaram que as prioridades competitivas de produção são definidas em função das estratégias competitivas (que refletem fatores de mercado como, por exemplo, demanda mais ou menos aquecida) e das características das áreas de decisão (que refletem os recursos acumulados e as pressões decorrentes de determinada trajetória). Estas características também podem ser verificadas na proposição obtida com o resultado da otimização multiobjetivo.

O resultado do modelo 1 evidenciou que, se a estratégia objetiva maximizar o lucro, então deve-se produzir a totalidade dos produtos no sistema MTO resultando num lucro de R $\$ 64.625$. Porém, se a empresa adotar esse modelo poderá haver prejuízo no atendimento aos consumidores que anseiam por maior disponibilidade do produto.

Olhager e Prajogo (2011) exploraram as diferenças entre os sistemas MTO e MTS em 216 empresas industriais australianas. Os autores encontraram diferença de foco entre as empresas que operam com MTO e MTS. No sistema MTO, as empresas têm impacto significativo, no seu desempenho, em relação a integração de fornecedores. Este impacto é menor para práticas de manufatura enxuta e racionalização de fornecedor.

Ainda de acordo com Olhager e Prajogo (2011), esta situação é revertida para as empresas que operam sob o sistema MTS, uma vez que este sistema tem efeito significativo relacionado a práticas internas de manufatura enxuta e racionalização de fornecedores, mas não para a integração logística com o fornecedor.

Os resultados da otimização multiobjectivo também permitiram propor uma solução equilibrada para a composição de programação destinada ao MTO e MTS. Historicamente, a empresa vinha produzindo $75 \%$ de produtos sob o sistema MTO e $25 \%$ sob o sistema MTS. Tal como pode ser verificado na Tabela 1, a solução do modelo 3 considerando os objetivos de maximizar o lucro e a produção pode resultar numa sugestão de proporção de $63 \%$ de MTO e $37 \%$ de MTS. 
ALVES FILHO, A. G.; NOGUEIRA, E.; BENTO, P. E. G. Análise das estratégias de produção de seis montadoras de motores para automóveis. Gestão \& Produção, São Carlos, v. 3, n. 18, p.603-618, 2011.

ARENALES, Marcos et al. Pesquisa operacional: para cursos de engenharia. Elsevier Brasil, 2015.

CORDEIRO, J. C. A.; ASSUMPÇÃO, M. R. P. Sistemas híbridos de produção: produzir para estoque ou para atendimento a pedidos? Revista de Ciência e Tecnologia, v.17, n. 34, p. 113-124, jan./jun., 2014.

DASSAN, E. F. ; SANTOS, D. ; SILVA, A. M. ; KAWAMOTO JUNIOR, L. T. ; RODRIGUES, E. F. Otimização multiobjetivo em uma Linha de Produção de Placas Eletrônicas de uma Pequena Empresa. Espacios (Caracas), v. 37, p. 5, 2016.

FÁVERO, Patrícia Belfiore; ZOUCAS, Fabio Augusto Mollik. Redes neurais para previsão da produção industrial de diferentes segmentos. Produto \& Produção, v. 17, n. 2.

GUIMARÃES, A. A. Utilização de uma abordagem híbrida System Dynamics-Factory Physics para a investigação do efeito de ações de melhoria contínua na redução do lead time. 2010. 205 f. Dissertação (Mestrado) - Curso de Engenharia de Produção, Ufscar, São Carlos, 2010.

HOPP, W. H. ; SPEARMAN, M. L. Factory physics: foundations of manufacturing management. 2. ed. New York, Ny, Usa: Mc Graw-hill, 2000. 726 p.

JABBOUR, A. B. L. S.; ALVES FILHO, A. G.. Tendências da área de pesquisa em estratégia de produção. Revista Eletrônica Sistemas \& Gestão, São Carlos, v. 3, n. 4, p.238-262, maio 2010

KALANTARI, M.; RABBANI, M.; EBADIAN, M.. A decision support system for order acceptance/rejection in hybrid. Applied Mathematical Modelling, Tehran, 21 set. 2010. p. 13631377.

KAMINSKY, Philip M.; KAYA, Onur. MTO-MTS Production Systems in Supply Chains. NSF Design, Service, and Manufacturing Grantees and Research Conference, St. Louis, Missouri. 2006.

LACHTERMACHER, G. Pesquisa Operacional: na tomada de decisões. 4. ed. São Paulo: Pearson, 2009.

MARINS, F.A.S. Introdução a pesquisa Operacional. São Paulo: Cultura Acadêmica, 2011.

MARTINS, G.A. Estudo de caso: uma reflexão sobre a aplicabilidade em pesquisas no Brasil. Revista de Contabilidade e Organizações. v. 2, n. 2, p.8-18, janeiro a abril de 2008.

MIGUEL, P. A. C. (Coord.). Metodologia de Pesquisa em Engenharia de Produção e Operações. 2. ed. Rio de Janeiro: Elsevier, 2012.

OLHAGER, J.; PRAJOGO, D. I. The impact of manufacturing and supply chain improvement initiatives: A survey comparing make-to-order and make-to-stock firms. Omega, Sweden, p. 159-165. 13 maio 2011.

PAIVA, E. L.; CARVALHO JUNIOR, J. M.; FENSTERSEIFER, J. E. Estratégia de produção e de operações: conceitos, melhores práticas, visão de futuro. 2ed. Porto Alegre, RS: Bookman, 2009. 253p.

PIO, A. ; SILVA, A. M. ; KAWAMOTO JUNIOR, L. T. . Otimização da produção em sistemas puxados: análise em uma fábrica de móveis de aço. Revista Científica Hermes, v. 20, p. 62, 2018.

RAGSDALE, C. T. Modelagem e Análise de Decisão. São Paulo: Cengage, 2009.

SHAMBLIN, J. E. ;STEVENS JUNIOR, G. T. Pesquisa Operacional: Uma abordagem básica. $1^{\text {a }}$ Edição São Paulo: Atlas, 1979. 426 p. 
SLACK, N.; CHAMBERS, S.; JOHNSTON, R. Operations Management. 5. ed. São Paulo: Saraiva, 2007.

WANROOIJ, M.R. Strategic supply chain planning in a multi-echelon environment: Identification of the CODP location constrained by controllability and service requirements School of Industrial Engineering. Series Master Theses Operations Management and Logistics Eindhoven, August 2012.

YIN, Robert K. Estudo de Caso, planejamento e métodos. 2.ed. Sao Paulo: Bookman, 2001. 